\title{
¿Con ventanas o sin ventanas? Winch, Apel y la monadología de las formas de vida*
}

\author{
Gonzalo Scivoletto**
}

\begin{abstract}
Resumen
El siguiente trabajo se propone analizar algunas categorías epistemológicas a partir del problema antropológico de comprender una forma de vida "extraña". Para ello, se toma como hilo conductor el programa filosófico-social de Peter Winch y en particular su crítica a la obra clásica de la antropología "Brujería, oráculos y magia entre los azande" de Evans-Pritchard. Winch, siguiendo a Wittgenstein, representa un verdadero cambio de paradigma dentro de la tradición analítica de las ciencias sociales, el cual muestra algunas similitudes con la hermenéutica (Gadamer, Ricoeur), la filosofía intercultural (Fornet-Betancourt) y el pragmatismo (Bernstein). De particular importancia resulta en este contexto el problema de la multiplicidad de las formas de vida y de la respectiva "conmensurabilidad" de los estándares de racionalidad. En respuesta a la acusación de "relativismo" de su propuesta teórica, puede afirmarse que en Winch hay dos categorías de análisis antropológico-social que permitirían, o que orientarían, la comprensión intercultural: las analogías formales y las nociones limitantes. Finalmente, se contrastan tales categorías con las críticas de Karl-Otto Apel.

Palabras clave: interculturalidad, antropología, relativismo, reglas, formas de vida.
\end{abstract}

\begin{abstract}
The following paper analyzes some epistemological categories from anthropological problem of understanding a "strange" form of life. To do this, it is taken the philosophical social program of Peter Winch and in particular his critique of classic anthropology "Witchcraft, Oracles
\end{abstract}

\footnotetext{
* Recibido: mayo 2016. Aceptado: junio 2016.

** Universidad Juan Agustín Maza. Mendoza, Argentina. Email: scivolettog@gmail.com
} 
and Magic among the Azande" by Evans-Pritchard. Winch, following Wittgenstein, represents a true paradigm shift within the analytic tradition of social science, which shows some similarities with hermeneutics (Gadamer, Ricoeur), philosophy and intercultural ethics (Fornet-Betancourt) and pragmatism (Bernstein). In this context, the problem of the multiplicity of forms of life and the respective "commensurability" of standards of rationality it is taken. In response to the charge of "relativism" from his theoretical proposal, it can be said that there are two categories of anthropological and social analysis that would allow or that would guide intercultural understanding: formal analogies and limiting notions. Finally, these categories are contrasted with the perspective of Karl-Otto Apel.

Key words: interculturality, anthropology, relativism, rules, forms of life.

\section{Peter Winch y la filosofía social}

Hay dos ideas clave que nos permiten ingresar en el pensamiento de Winch, y que funcionan a su vez como una superación interna de la filosofía analítica positivista. En primer lugar, el rechazo a lo que él denomina "concepción subordinada" de la filosofía (propia del positivismo), y en segundo lugar la asunción del giro lingüístico (en el sentido del segundo Wittgenstein). La "concepción subordinada" es aquella que sostiene que la filosofía debe estar al servicio de la ciencia limpiando los obstáculos que impedirían el avance del progreso científico, en la medida que esos obstáculos son de índole lógico-conceptual. De acuerdo con ello, la filosofía no se ocuparía de "realidades" sino de "conceptos" y por lo tanto no sería más que una "técnica para resolver los problemas planteados en el curso de investigaciones no filosóficas" (Winch, 1990: 12). De tal modo que, por ejemplo, campos filosóficos como la metafísica y la epistemología deberían ser comprendidos como antesalas al verdadero conocimiento (por ejemplo, el que desarrolla la psicología, la economía, la sociología, etc.), o bien, como un momento de autoexamen para perfeccionar las "herramientas" propias de la filosofía y retornar de manera más aclarada a su "auténtica" tarea: el análisis del lenguaje científico. En conclusión, desde esta concepción, la filosofía no puede hablar de la realidad puesto que no cuenta con ella en sus proposiciones, ella es más bien, un instrumento u órganon de la ciencia.

Ahora bien, como señala correctamente Winch, a raíz de esta función que se le atribuye a la filosofía y la correspondiente división de trabajo con la ciencia, uno podría preguntarse qué entiende por "realidad" la propia concepción subordinada. ¿Es el mismo sentido de la palabra realidad aquel que subyace en el cientificismo que aquel que propone la filosofía (clásica o "no subordinada")? En primer lugar, se debe reconocer que una cosa es preguntarnos por cosas reales o que existen en la realidad y otra muy distinta es plantear la pre- 
gunta por la realidad misma. En este caso, como afirma el propio Winch, "uno puede representarse una lengua que no tenga el concepto de humedad, pero difícilmente una en la que no haya modo de distinguir lo real de lo no real", es decir que al investigar la realidad el científico ya está comprometido con algún concepto de realidad, de aquí que "una discusión sobre la naturaleza del significado de realidad parece tener una inevitable dimensión reflexiva" (Horton, 2000: 26) que, agregamos nosotros, el filósofo debe "sacar a la luz", precisamente, mediante la reflexión sobre el lenguaje. Ahora bien, luego de esta disquisición sobre el concepto de realidad, si observamos atentamente, de algún modo Winch también termina aceptando que la tarea de la filosofía consiste en una "elucidación conceptual". Sin embargo, el problema no está en que la filosofía se ocupe del lenguaje, sino en el énfasis o la perspectiva que se pone en ello. Es decir, "el filósofo no se interesa en el uso correcto [del lenguaje] como tal, ni todas las confusiones lingüísticas son igualmente relevantes para la filosofía. Sólo lo son en cuanto su análisis está destinado a esclarecer el grado de inteligibilidad de la realidad, y qué diferencia constituiría para la vida del hombre el hecho de que este pudiese aprehender en cierta forma dicha realidad" (Winch, 1990: 17). Por ello, la cuestión de la confusión lingüística no es relevante en sí, sino que lo fundamental es la relación entre el lenguaje y la realidad, y cómo el lenguaje "dice" el mundo. En otras palabras, lo que se está mostrando, a nuestro juicio, es que el interés central de la filosofía es la significación o constitución del sentido que se realiza en y por el lenguaje. Por ello, siguiendo a Wittgenstein, Winch puede afirmar: "El mundo es para nosotros lo que se manifiesta a través de conceptos. Esto no significa que nuestros conceptos no puedan cambiar; pero cuando lo hacen, nuestro concepto del mundo también cambia" (Winch, 1990: 21).

La filosofía, entonces, apunta a la comprensibilidad o inteligibilidad de la realidad, mediada por el lenguaje. ¿Pero qué significa esto? Winch advierte que hablar de "inteligibilidad" o "comprensibilidad" es bastante complejo, dado que se trata de conceptos ambiguos y que se utilizan de diferentes maneras en diferentes contextos. Muchas actividades humanas encaran tareas de "comprensión" de la realidad, no se trata sólo de una cuestión que atañe al filósofo o al científico. También la religión, la mitología o el arte son formas de inteligibilidad de la realidad. En términos de Wittgenstein, se trata de distintos juegos de lenguaje en los cuales la comprensión adquiere un sentido más o menos preciso. Las filosofías "periféricas", como las llama Winch, se ocuparían de las distintas formas de comprensión. Así, por ejemplo, una filosofía de la religión se abocaría a la tarea de comprender la comprensión del juego de la religión, una estética se ocuparía del juego de lenguaje del arte, etc. Ahora, en este caso, no se trata de una concepción subordinada de la filosofía dado que su interés es genuinamente filosófico y no es parasitario de la ciencia, la religión, el arte, etc. Pero tal concepción, como rápidamente puede apreciarse, implica una serie de cuestiones. La primera tiene que ver, nuevamente, con el concepto de comprensibilidad. Pues, si existen diferentes juegos de lenguaje en los que la comprensibilidad "se juega" de diversas maneras, ¿en qué consiste, en sí misma, la comprensibilidad a partir de la cual 
"reconstruimos" las distintas "comprensibilidades"? Segundo, ¿no se disuelve la filosofía en las múltiples empresas humanas? ¿Se puede elaborar una epistemología general que vaya más allá de los diferentes juegos de lenguaje? En síntesis, ¿podemos participar en un juego de lenguaje que hable de todos los juegos de lenguaje? La segunda pregunta nos conducirá hacia el final del trabajo al planteo de Karl-Otto Apel y su idea de un juego trascendental del lenguaje. Pero antes intentaremos ver cómo Winch responde a la primera cuestión.

La categoría epistemológica que utiliza Winch para el análisis de las "comprensibilidades" es aquella que Wittgenstein llama "seguir una regla"1. Dado que el lenguaje es una práctica social (la práctica social por excelencia), también se puede ampliar el uso de dicha categoría hacia otras formas de interacción humana a partir de la idea de que se trata de "conductas significativas". Tarea que, como bien señala Apel, ya había sido encarada por Dilthey, pero en lugar de fundamentar las ciencias sociales apelando a la empatía (Einfüllung), Winch lo hace según la mencionada categoría wittgensteiniana. Veamos primero qué debemos entender por conducta significativa. Winch menciona el siguiente ejemplo: un individuo $\mathrm{N}$ vota a un determinado partido político X por la razón R. Esta razón o motivo es un fundamento para la acción. Pero si ahora agregamos un observador $\mathrm{O}$ que explica la conducta de $\mathrm{N}$ por la razón $\mathrm{R}$, estamos obligados a pensar que los conceptos contenidos en la explicación E deben ser comprendidos por O. Siguiendo el ejemplo, si la razón R es que "asegura el pleno empleo", entonces O debe conocer el concepto de "pleno empleo" como fuerza motivacional de la acción de N. Pero no sólo eso, sino que $\mathrm{N}$ mismo debe comprender dicho concepto, pues no tendría sentido decir que $\mathrm{N}$ votó al partido X por tal razón R. Aunque sólo el "experto" (por ejemplo, un economista) puede explicar detalladamente el concepto "pleno empleo", $\mathrm{N}$ "sabe" que se trata de que "todos tengan trabajo". Por ello, como en el psicoanálisis, el paciente debe participar de la explicación brindada por el analista. En nuestro ejemplo, un economista podría preguntarle a $\mathrm{N}$ qué quiere decir con su expresión "que todos tengan trabajo" y luego aquel puede decirle: "efectivamente, lo que usted describe, en economía se denomina 'pleno empleo'". Es decir, la comprensión de la conducta supone necesariamente el punto de vista subjetivo, pero como un momento necesario en la justificación de la validez de dicha comprensión. Ahora bien, ¿qué sucedería en el caso de un individuo que vota a un partido sin ninguna razón o motivo? En tal caso, uno estaría tentado a pensar que tal acción no tiene sentido. Sin embargo, no se trata de cualquier acción, se trata de sufragar, lo cual, está enmarcado dentro de una práctica social o forma de vida. Con ello, desde nuestro punto de vista, Winch intenta romper con el sentido

\footnotetext{
${ }^{1}$ No podemos ingresar aquí en el tratamiento de Wittgenstein de esta cuestión, la cual se pueden encontrar aproximadamente en los parágrafos 138-242 de las Investigaciones filosóficas, ni tampoco en la discusión erudita, sobre todo a partir de la interpretación de Kripke. Para una visión general remitimos a Corredor, 1999: 384 ss. Lo que se intenta mostrar aquí es el uso que realiza Peter Winch de esta y otras categorías provenientes de la tradición de Wittgenstein.
} 
"mentalista" o "subjetivista" de la acción significativa para enmarcarla en el todo social, esto es, como una práctica. Analizar una conducta significativa es estudiar las reglas que se siguen en dicha conducta o práctica y no "adivinar" la intención subjetiva del actor.

Ahora bien, para comprender la noción de "regla", podemos apelar a la distinción con el "hábito". En principio, un hábito es algo que se aprende y se practica sin modificación. Wittgenstein utiliza el ejemplo del aprendizaje de los números naturales: aprender a desarrollar la serie de estos números no significa copiar y repetir lo que el maestro ha escrito en el pizarrón, sino aprender el criterio para realizarlo, es decir, saber cuándo es correcto o no seguir la serie de determinada manera, y la posibilidad de aplicarla a diferentes situaciones. Un perro puede ser condicionado para que actúe de cierta manera, es decir, puede desarrollar cierto hábito, "mientras que yo sé la forma correcta de continuar sobre la base de lo que se me ha enseñado" (Winch, 1990: 61). Ese "saber" alude sin duda a la capacidad de reflexividad que supone la conducta humana a diferencia de los animales. Ello entraña una segunda característica del "seguir una regla": la posibilidad de no seguirla, es decir, tener una alternativa y la oportunidad de fallar. Dice Winch: "Comprender algo implica también comprender lo opuesto: yo comprendo lo que es actuar en forma honesta justo en la medida en que comprendo lo que es actuar deshonestamente. He aquí por qué la conducta que es producto de la comprensión, y solo esa conducta, es aquella para lo cual existe una alternativa" (Winch, 1990: 64). Por último, hay una tercera característica del seguir una regla. Se trata de la fundamental y tal vez más conocida característica: nadie puede, solo, seguir una regla, es decir, un lenguaje privado es imposible. Ello implica dos cosas, primero que las reglas están entrelazadas en contextos de formas de vida, y segundo, que cualquiera que quiera entender dichas reglas debe poder participar de tal forma de vida. En conclusión, una conducta significativa será aquella que se caracterice por el seguir una regla, y ello supone tres requisitos: poder reflexionar sobre la regla, comprender que se sigue una regla y poder idear formas alternativas de aplicación.

\section{El problema de una monadología de las formas de vida}

Los grupos, culturas o formas de vida se autoconstituyen o se autodefinen en un proceso incesante que presupone siempre una relación con otros grupos, culturas o formas de vida a los que, a su vez, se los "categoriza" de una determinada manera ${ }^{2}$. Cada forma de vida posee un background de creencias, valores, prácticas a partir de las cuales se orienta la praxis cotidiana y se determina "lo que es el caso". El contacto entre dos formas de vida, grupos o culturas puede provocar la colisión entre tales creencias, valores o prácticas y

\footnotetext{
${ }^{2}$ La expresión "formas de vida" (Lebensformen) posee la ventaja de no reducir los grupos a sus características étnicas. Por su parte, el concepto de cultura ha resultado problemático incluso para la misma filosofía intercultural (ver Viaña, 2009).
} 
conducir a uno de los grupos (o a ambos) a poner en marcha ciertos modos de recomposición del "malentendido" o del "desacuerdo". El problema que se presenta aquí es en qué medida o hasta qué punto es posible la inter-comprensión cuando los estándares de racionalidad son radicalmente diferentes. Probablemente hoy en día en las ciencias sociales y en la filosofía ya (casi) nadie esté dispuesto a negar la raigambre histórico-cultural, esto es, relativa a un mundo de vida, de posicionarse y orientarse en el mundo. Incluso a primera vista uno estaría en condiciones de asentir a la afirmación de Panikkar: "Cada cultura es una galaxia que alberga la experiencia y la percepción del mundo a partir de los cuales surge la autocomprensión, las preguntas que distinguen una cultura de otra y que definen lo que es significativo para una colectividad: los criterios de verdad, de bondad, de belleza, así como los límites del mundo y la manera de posicionarse" (Panikkar, 2006: 129). Ahora bien, la idea de las culturas como galaxias que contienen la totalidad de la experiencia, esto es, como mónadas, no parece dejarnos otra opción que la de preguntarnos si tales mónadas tienen o no "ventanas". En todo caso, la pregunta es cómo es posible (si lo es) trascender el propio mundo de vida, la "mónada" o "galaxia" en la que somos, juzgamos y valoramos.

Para este problema, el breve ensayo de Peter Winch "Para comprender una sociedad primitiva", puede servir de orientación. Aquí Winch reflexiona sobre las condiciones de posibilidad de comprender un mundo de vida radicalmente diferente a partir de la lectura del clásico de la antropología Brujería, oráculos y magia entre los azande de Evans-Pritchard (1937).

Winch reconstruye la situación ante la que se encuentra el antropólogo en la investigación sobre las prácticas mágicas y oraculares de los azande:

La tarea del antropólogo que estudia estos pueblos para hacer inteligibles tales creencias y prácticas tanto para sí como para sus lectores, significa presentar una explicación de ellas que satisfaga, de alguna forma, el criterio de racionalidad demandado por la cultura a la que él y sus lectores pertenecen; una cultura cuya concepción de racionalidad está profundamente afectada por los logros y métodos de las ciencias, y que asume las creencias en la magia o las prácticas de consultar a los oráculos casi como paradigma de lo irracional. Las tensiones inherentes a esta situación muy probablemente lleven al antropólogo a adoptar la siguiente postura: sabemos que las creencias azande sobre la influencia de la brujería, la eficacia de las medicinas mágicas, el papel de los oráculos para revelar lo que está pasando y lo que sucederá, son erróneas, ilusorias [...] Todo lo que podemos hacer, entonces, es mostrar cómo un sistema de creencias erróneas y de prácticas ineficaces puede mantenerse a sí mismo frente a objeciones al parecer tan obvias (Winch, 1991: 82).

\footnotetext{
${ }^{3}$ En este contexto entendemos el "malentendido" como una "incomprensión" respecto del sentido, susceptible de ser resuelta, en principio, mediante un discurso explicativo (Schnädelbach). En cambio, llamamos "desacuerdo" a una "postura diferente" respecto de un hecho (discurso teórico) o de una norma (discurso práctico).
} 
En la cita anterior subyace una distinción respecto de la cual Winch considera que Evans-Pritchard "está totalmente equivocado". Nos referimos a la distinción entre lo lógico y lo científico. Veamos el texto de Evans-Pritchard.

Nociones científicas son aquellas que concuerdan con la realidad objetiva tanto en lo que se refiere a la validez de sus premisas como a las inferencias que se sacan de sus proposiciones [...] Nociones lógicas son aquellas en que de acuerdo con las reglas del pensamiento, las inferencias serían verdaderas si las premisas lo fueran, siendo irrelevante la verdad de las premisas [...] se ha roto una olla durante la cocción al fuego. Tal vez se deba a que tenga polvo. Examinemos la olla y veamos si es ésa la causa. Esto es pensamiento lógico y científico. La enfermedad se debe a la brujería; un hombre está enfermo; consultemos a los oráculos para descubrir quién es el brujo responsable; esto es pensamiento lógico pero acientífico (cit. en Habermas, 1999: 86).

El antropólogo, que debe satisfacer los criterios de racionalidad de su propio mundo de vida o cultura, debe encargarse de comprender la comprensión global del mundo de vida "primitivo" desde el punto de vista de su consistencia o coherencia interna, y no así, respecto de, podríamos decir, el contenido semántico o el plano referencial de tales creencias. Pues "claramente", las creencias de los azande "no se corresponden con la realidad". Este es el punto de discusión. Lo problemático parece ser entonces una cuestión filosófica acerca de la relación entre lenguaje y realidad, y no solamente una cuestión empírica (qué sucede "de hecho", etc.).

La realidad no es lo que le da sentido al lenguaje. Lo real y lo irreal se manifiestan en el sentido del lenguaje. Además, tanto la distinción entre lo real y lo irreal como el concepto de concordancia con la realidad pertenecen a nuestro lenguaje. No diré que sean conceptos del lenguaje como otros, pues queda claro que ahí ocupan una posición dominante y en cierto sentido limitante. Podemos imaginar un lenguaje sin concepto de humedad, digamos, pero difícilmente uno en el que no hubiera forma de distinguir lo real de lo irreal. Sin embargo no podríamos diferenciar lo real de lo irreal sin entender la manera como opera esta distinción en el lenguaje. Si deseamos entonces entender el significado de estos conceptos, debemos examinar el uso que de hecho tienen en el lenguaje (Winch, 1991: 84).

Winch, siguiendo a Wittgenstein, entiende que el lenguaje se encuentra entrelazado con la forma de vida. Cada cultura o forma de vida "abre" una determinada realidad, los "límites del lenguaje son los límites del mundo", es decir que "la" realidad es inmanente al lenguaje. El problema con el enfoque de Evans-Pritchard, según Winch, es que este no se "limita a registrar" los diferentes lenguajes y realidades de la concepción científica y de la concepción mágica del mundo, sino que, en última instancia, cree que la concepción científica efectivamente concuerda con la realidad y la mágica no. Sin embargo, hay todavía un problema mayor, que consiste en lo que Winch denomina "error categorial" del antropólogo. El punto de partida es la observación 
de que frente al señalamiento de inconsistencias o contradicciones entre los oráculos, los azande, que manifiestan "cierta incomodidad", prefieren, por decirlo así, abandonar el asunto.

Vale la pena observar... que los azande, cuando se les señala la posibilidad de esta contradicción en relación con la herencia de la brujería, no llegan a considerar entonces obsoletas sus viejas creencias en la brujería. "No tienen ningún interés teórico en la materia" [E.-P.]. Esto permite sospechar que el contexto desde el cual se hace la sugerencia acerca de la contradicción, el contexto de nuestra cultura científica, no está en el mismo nivel que el contexto en el cual operan las creencias en relación con la brujería. Las nociones azande de la brujería no constituyen un sistema teórico en términos de obtener un conocimiento cuasi-científico del mundo. Esto a su vez sugiere que el europeo, obsesionado en presionar el pensamiento azande hacia donde naturalmente no iría -a una contradicción-, es el culpable del malentendido, y no los azande. El europeo está incurriendo, de hecho, en un error de categoría (Winch, 1991: 90).

El error categorial, que ocasiona el malentendido, puede analizarse en dos sentidos, igualmente conectados. Por un lado, considerar las prácticas de la brujería, magia y consulta de oráculos como formas primitivas del conocimiento científico, en el sentido moderno cognitivo-instrumental. Por otro, creer que la actitud de los azande hacia las propias creencias es propia de una "mentalidad cerrada" (Horton) ${ }^{4}$, esto es, que no está dispuesta a ensayar otras alternativas de interpretación, lo que se contradiría con el espíritu moderno de la crítica, como la actitud o la capacidad de poner a prueba siempre y constantemente las propias creencias. No obstante, el problema es, como afirma Winch, que

[...] nosotros no tenemos inicialmente una clasificación que se parezca ni remotamente a la categoría azande de magia. Y puesto que queremos entenderla, tenemos la obligación de ampliar nuestro modo de comprender, de tal manera que le abramos un espacio a la categoría azande, en lugar de insistir en verla en términos de nuestra propia distinción preestablecida entre ciencia y no-ciencia. Ciertamente, la clase de comprensión que buscamos exige que veamos la categoría azande en relación con nuestras propias categorías ya sobreentendidas (Winch, 1991: 95).

Aquí aparecen dos elementos a destacar. En primer lugar, que si para comprender es necesario participar del juego de lenguaje o mundo de vida, entonces resulta difícil explicar cómo se puede comprender por fuera de tal mundo. Como intenta mostrar Winch, la comprensión de la magia en la cultura azande sólo puede llevarse a cabo por medio de analogías con "nuestras" propias prácticas. Pero esto es, más bien, un proceso de ensayo y error, un proceso que al mismo tiempo nos pone en la tarea de autocomprendernos

\footnotetext{
${ }^{4}$ Para un análisis crítico de esta perspectiva, véase Habermas, 1999: 93 ss.
} 
para comprender a los otros, y viceversa. En este sentido, en lugar de interpretar la magia como una técnica instrumental, Winch propone o ensaya establecer una analogía entre las prácticas mágicas y la práctica cristiana de la oración. Quien reza o suplica "hágase Tu voluntad" no está utilizando un medio para conseguir tal o cual fin, por ejemplo que llueva, sino que, según Winch, expresa un modo de ser o de actuar frente a las eventualidades. Es decir, no cabría aquí hablar de "racionalidad instrumental", sino que la magia podría ser comprendida no como una práctica que busca dominar y controlar los acontecimientos (pues esto sí sería una forma de proto-ciencia) sino una forma de reconocer nuestra vulnerabilidad y entrega a ellos, esto es, nuestra finitud e impotencia.

La posición de Winch representa una forma de pluralismo intercultural, según el cual, los criterios de racionalidad, belleza, utilidad, veracidad, relación a la facticidad, entre otros, son inmanentes a cada forma de vida. Sin embargo, Winch no niega la posibilidad de la inter-comprensión o de la crítica intercultural, algo que por otra parte sería poco serio defender, pues la historia de la humanidad no consiste más que de las "críticas" entre distintas formas de vida. La advertencia de Winch está dirigida contra la tendencia de la ciencia o de, por decirlo así, "nuestra" forma de vida a absolutizar nuestros propios criterios de racionalidad. Pero además de esta recomendación ética, por así decir, hay en la filosofía de Winch un esbozo epistemológico acerca de la posibilidad de evitar el relativismo, es decir, de la absolutización u ontologización de la diferencia.

\section{Apel con Winch y contra Winch}

La reflexión de Karl-Otto Apel sobre Winch se inscribe en el contexto de una reconstrucción histórica de la filosofía analítica (Apel, 1985 II: 27-90). Dicha tarea se realiza tomando como hilo conductor un concepto, en principio, externo a esta tradición, esto es, el concepto de comprensión tal como aparece en la tradición hermenéutica de las ciencias del espíritu. Para Apel, la filosofía analítica puede dividirse en tres etapas: atomismo lógico, positivismo lógico y filosofía analítica del lenguaje. Es precisamente en esta última etapa donde convergen de manera sistemática los planteos continentales, principalmente de Heidegger y Gadamer, y los analíticos, fundamentalmente Wittgenstein y, en este caso, Winch. Por cuestiones de espacio, iremos directamente al último punto.

Según Apel, el pensamiento de Winch puede ser entendido como un pensar con Wittgenstein contra Wittgenstein (Apel, 1985 II: 68 ss.). Con Wittgenstein en el sentido de que él muestra cómo la identificación de objetos está entrelazada con reglas, y que las reglas, a su vez, están entretejidas con formas sociales de vida, y que, por último, seguir una regla está entrelazada con la posibilidad de reflexionar sobre dicha regla. Sin embargo, aquello en lo que, según Apel, Winch está en contra es la consecuencia que tal concepción tiene para la filosofía, entendiéndola no como un juego de lenguaje 
vacío, sino como el juego de lenguaje sobre la comprensión del sentido. En efecto, Wittgenstein, junto con la mayoría de los filósofos analíticos de aquella primera etapa, podría ser incluido en aquella concepción de la filosofía subordinada que se explicaba al comienzo. Ahora bien, la novedad y el aporte de Winch, según Apel, consiste en no reducir tal comprensión del sentido a la mera descripción empírica de formas de vida (interpretación conductista de Wittgenstein). La comprensión, entonces, se encuentra en una dimensión epistemológica que es anterior a todo conocimiento empírico. Y en este punto coincide el enfoque analítico con el de la filosofía hermenéutica de Heidegger y Gadamer. Ambas tradiciones rechazan la explicación psicológica, y con ello el solipsismo metódico, y, por lo tanto, comparten la orientación hacia el lenguaje como mediación del entendimiento y la interpretación del mundo. En efecto, "...en Winch, como en Heidegger, todo conocimiento supone ya una determinada comprensión del mundo acreditada públicamente en el ser unos con otros" (Apel, 1985 II: 79). Sin embargo, advierte Apel, la vieja tradición de las ciencias del espíritu buscaba comprender determinadas "objetivaciones del espíritu" (arte, religión, etc.) de seres humanos o grupos históricos de carne y hueso, y no (o no sólo) explicar o descubrir la pre-estructura comprensiva de la realidad. Es decir, se trata de una cuestión empírica y no filosófica, en la medida que queremos entender una cultura o forma de vida "extraña" a nosotros. Por ello, Apel se pregunta cómo despliega Winch la relación entre la comprensión en su sentido filosófico y la comprensión en su sentido empírico, dado que no es legítimo (y sería contrario al giro lingüístico) retornar a conceptos "psicológicos" como los de "empatía" (Dilthey) para comprender formas de vida ajenas. Para ello, debemos detenernos un momento en la concepción que tiene Winch de la actividad del sociólogo (en sentido amplio), contrastada con el proceder del científico natural.

En el prefacio a la segunda edición de The Idea of Social Sciences and It's Philosophy, del año 1990, el propio Winch señala dónde se encuentra el núcleo de su propuesta teórica (Winch, 2003: IX ss). El argumento es más o menos como sigue: pensemos en una persona que no conoce sobre física nuclear y se encuentra frente al experimento de Cockroft-Walton, que consiste en el bombardeo de hidrógeno sobre litio; en tal caso, la descripción misma del experimento le resultaría incomprensible al neófito, dado que no conoce lo que hacen los físicos nucleares, y ello incluye el significado del término "bombardeo" en este ámbito. Las reglas que siguen los físicos nucleares "se apoyan en un contexto social de actividad común" (Winch, 1990: 81), por lo que comprender la actividad del científico implica conocer su relación con el fenómeno que investiga y, además, su relación con los colegas, es decir, su pertenencia a una comunidad de comunicación. Por ello, el fenómeno que estudia el científico natural posee, por decirlo así, un estatus diferente al del sociólogo, puesto que en el caso del fenómeno social, según Winch, se requiere algún tipo de participación en el juego de lenguaje que se intenta comprender. Esto puede ser ilustrado con otro ejemplo. Imaginemos una situación en la cual un hombre le arroja agua en la cabeza a un niño. Para que un observador-investigador social describa este hecho como un "bautismo", 
dicho investigador debe "participar"s, de algún modo, de los ritos de iniciación religiosa. Es decir, un observador que no conoce la práctica social denominada bautismo, no puede comprender la escena como bautismo. De ello, Winch extrae una consecuencia que para Apel resultará problemática: "la tarea peculiar de la filosofía consiste en adoptar un criterio no comprometido de tales concepciones [juegos de lenguaje]; su misión no es otorgar premios a la ciencia, la religión, o cualquier otra cosa. Tampoco lo es defender ninguna Weltanschauung. En términos de Wittgenstein 'la filosofía deja todo como está"” (Winch, 1990: 97).

Ahora bien, la pregunta que no se puede dejar de plantear es la siguiente: Si los juegos lingüísticos están ya dados como formas últimas de comprensión del sentido, ¿desde qué lugar se realiza la descripción de los juegos de lenguaje mismos? Es decir, pareciera que hay un juego de lenguaje que se refiere a la totalidad de los juegos de lenguaje, del cual, en tanto juego de lenguaje, debemos poder participar, pues de lo contrario recaeríamos en el objetivismo cientificista que cree ingenuamente que puede realizarse una descripción neutral, distanciada de la sociedad como comunidad de comunicación. Por lo tanto, paradójicamente, el relativismo que parece defender Winch coincide con el objetivismo que combate, puesto que la filosofía "debe dejar todo como está" y, al mismo tiempo, describir de "modo no comprometido". Nos encontramos atrapados en un círculo: o estamos dentro, y no hay posibilidad de la distancia crítica, o estamos fuera, y por lo tanto no podemos describir nada.

Según Pleasants (2000: 79 ss.), hay cuatro objeciones principales que se han planteado a la filosofía social de Winch: 1) reducir la ciencia social a filosofía; 2) mantener una postura idealista, en el sentido de no considerar la base material de la sociedad; 3) su concepción epistemológica para las ciencias sociales, ya que "no permite otra forma de explicación que vaya más allá de la auto-comprensión de los participantes en un modo de vida social" (Pleasants, 2000: 80); 4) excluir cualquier forma de evaluación crítica (relativismo). Esta última objeción es, sin duda, la más profunda y la que nosotros quisiéramos abordar a continuación.

De acuerdo a lo que hemos analizado hasta aquí, todo parece concluir en que desde los presupuestos filosóficos de Winch (en continuidad con Wittgenstein) no es posible la evaluación crítica entre formas de vida extrañas. Los distintos Lebenswelten (expresión que el propio Winch utiliza en "Can We Understand Ourselves?") se comportan como Weltanschauungen o paradigmas "inconmensurables" (Pleasants, 1999: 35). Por lo tanto, si no es posible la "comparación", tampoco sería posible una evaluación crítica (critical assessment) entre paradigmas y, por ende, nuestro autor caería sin

\footnotetext{
${ }^{5}$ Apel lo formula de la siguiente manera: "Ahora bien, la multiplicidad y diversidad de las acciones humanas, no se pueden por ejemplo ni siquiera identificar y describir como objetos, sin el recurso a la comprensión de intenciones y de reglas que también uno mismo podría haber tenido, o podría haber seguido" (Apel, 2009: 216).
} 
más dentro de la etiqueta del relativismo, tal como lo interpreta Apel. Sin embargo, lo cierto es que el propio Winch rechazó firmemente tal acusación (Winch, 1972: 3; Brandon, 1982: 215), y algunos textos posteriores muestran un intento de salida del relativismo, aunque, desde nuestro punto de vista, no lo logre del todo.

Según Brandon (1982: 236 ss.), Winch intenta resolver el problema del relativismo en dos etapas: la primera mediante lo que él denomina "formal analogies" y luego las "limiting notions".

\subsection{Analogías formales}

Según Winch, entre todas las sociedades humanas deben existir algunas "similitudes", si bien no de contenido, sí de forma o función, en la medida que, por definición, toda sociedad posee un lenguaje. En efecto, como citamos anteriormente, si bien podemos representarnos una sociedad que no tenga el concepto de "humedad", no podríamos imaginar una sociedad como tal sin concepto de "realidad", aunque el contenido de este concepto varíe. En "Nature and Convention", Winch se refiere a la veracidad (truthfulness) como una "virtud moral", esto es, como "una condición de fondo (background) necesaria en cualquier sociedad en la que es posible para alguien hacer enunciados verdaderos" (Winch, 1972: 63). Una sociedad sin una norma de veracidad sería "autocontradictoria". Pero otro tanto se dice respecto de los conceptos de "realidad" (como ya mencionamos) y del concepto de "racionalidad".

La racionalidad no es sólo un concepto como otro cualquiera; debe también obedecer a un uso convencional, pero no es un concepto contingente (como el de cortesía, por ejemplo) sino necesario en cualquier idioma; decir que una sociedad tiene una lengua es decir que tiene un concepto de racionalidad. Tal vez no deba existir necesariamente en el otro lenguaje una palabra que signifique lo mismo que para nuestro término racional, mas, por lo menos, debe haber rasgos de un uso análogo al nuestro en relación con la palabra "racional" (Winch, 1991: 93-94).

Para Winch, sin embargo, la dificultad se mantiene, dado que "las reglas de la racionalidad" que pertenecen a mi mundo de vida no necesariamente pertenecen a la del otro, es decir, el problema consiste en la posibilidad del "salto" de un paradigma a otro. En este sentido, lo que Winch propondría, de manera cercana a la "fusión de horizontes" de Gadamer, es la ampliación de la categoría de racionalidad y de inteligibilidad mediante el aprendizaje y la apertura hacia la otra forma de vida. Por ello, las "analogías formales" (lenguaje, racionalidad, veracidad) funcionarían como una especie de "brújula" que le permitiría al investigador social "orientar la mirada", pero finalmente: "para estudiar otra forma de vida es necesario ampliar la nuestra -no simplemente incorporar la otra forma dentro de los límites ya existentes de la nuestra, porque el punto acerca de esta última, en su situación actual, es que sus límites ex hipothesi excluyen otras formas" (Winch, 1991: 93). Por otra parte, 
este "aprender unos de otros", por decirlo así, está orientado en el sentido de la "sabiduría" (Winch, 1991: 97), es decir, dicho en otros términos, el conocimiento de otra forma de vida no está orientado hacia la "manipulación", como en la ciencia objetivante, sino a un mejoramiento de la vida.

\subsection{Nociones limitantes}

La otra estrategia ya no es formal sino "material". Se refiere a "hechos" que pueden funcionar como elementos en común o "concordancias" (commonalities) entre las diferentes formas de vida. Esencialmente se trataría de aquellas situaciones en las que "están involucradas ineludiblemente en la vida de cualquier sociedad conocida, de forma tal que nos da una pista de dónde buscar si nos intriga el sentido de un extraño sistema de instituciones" (Winch, 1991: 98). Nuevamente se presenta aquí la idea de "pista" (clue), que funciona de algún modo como la orientación sobre la que recaerá la comprensión significativa descripta anteriormente.

Las "nociones" que Winch considera comunes son: el nacimiento, la muerte y las relaciones sexuales. Estas nociones son además "limitantes" porque, según Winch, "configuran" la vida humana. En efecto, la vida no se refiere simplemente a la existencia biológica, sino que dentro de esta categoría forman parte las preguntas por el sentido de la vida, la manera correcta de vivir, las cosas importantes en la vida, etc. Otro tanto sucede con la muerte, que tampoco es el mero dejar de existir: la muerte representa el "término de mi mundo", y "la actitud frente a la vida de uno es al mismo tiempo una actitud frente a la vida como algo que finaliza con la muerte de uno" (Winch, 1991: 99). Por último, el sexo configura el modo de ser de la vida humana, en tanto masculinidad o femineidad. Estas "ideas" o "nociones" conforman el espacio o la situación en la que se desenvuelven las acciones, por lo tanto, tienen un valor muy importante para la comprensión de tales acciones y para la ética.

Ahora bien, ambos modos de orientar la empresa de la comprensión no son "métodos", no ofrecen una garantía de comprensión (que es lo que ofrecería un método). Es decir que, finalmente, el resultado de las reflexiones de Winch es en cierto sentido escéptico. En uno de sus últimos textos, retoma la cuestión que había abordado más de 30 años atrás en "Understanding", el problema de la comprensión de las prácticas mágicas y oraculares de la tribu azande, pero la conclusión parece ser la misma: “... hay un tipo de comprensión de esta práctica [consulta del oráculo] que nosotros todavía no tenemos... nosotros no podemos imaginar la consulta del oráculo seriamente, como hacen los azande" (Winch, 1997: 199). El único camino posible resulta, entonces, una suerte de disposición al diálogo, a la "posibilidad de que el otro pueda tener razón" (Gadamer), pero, paradójicamente, con la garantía de que nunca podremos comprender cabalmente el sentido del otro debido a la pertenencia a distintas formas de vida. Por lo tanto, desde esta perspectiva, para una forma de vida secular o laicista, por ejemplo, resultaría muy difícil comprender o tomar seriamente una forma de vida basada en un libro sagrado 
o en preceptos religiosos como es el caso del Corán. Como dice Wittgenstein: „Ich kann mich in sie nicht finden". Pero precisamente en este punto nos encontramos con el gran aporte crítico de la ética discursiva de Apel. En efecto, si, como muestra el propio Winch, para la posibilidad de la comprensión de una cultura extraña debe tomarse en cuenta la propia comprensión de los sujetos estudiados, entonces, ello implica que deben ser tratados como "interlocutores discursivos", es decir, como iguales y co-responsables ${ }^{6}$.

Winch podría haber llegado a este punto de haber continuado en la línea de las "analogías formales". Así pues, en un pasaje de "Nature and Convention" sostiene: "si per absurdum el acaecimiento de enunciados "verdaderos" y "falsos" fuera estadísticamente aleatorio (random), entonces no podría haber ninguna distinción entre verdad y falsedad, por ende no habría comunicación" (Winch, 1972: 62). Es decir, no es lo mismo este concepto de veracidad que los conceptos científicos (o de cualquier esfera de la cultura), dado que uno puede imaginarse sociedades sin ciencia y sin conceptos tales como "átomo", "enzima" o "pragmática trascendental"; pero sin veracidad, no habría sociedad. Precisamente los estándares o parámetros de enjuiciamiento intercultural no pueden buscarse en las "concordancias" empíricamente observadas, sino en aquello que hace posible, trascendentalmente, cualquier forma de vida, o, expresado más técnicamente, las meta-reglas que hacen posibles las convenciones regidas por reglas y permiten la reflexión crítica sobre las reglas. En efecto, como afirma Apel:

[...] lo común entre todos los juegos lingüísticos radica, a mi juicio, en el hecho de que con el aprendizaje de un lenguaje -es decir, con la efectiva socialización de una forma de vida entretejida con el uso del lenguaje- se aprende algo así como el juego lingüístico, es decir, la forma humana de vida; se adquiere básicamente la competencia para reflexionar sobre el propio lenguaje o forma de vida y para comunicarse con todos los demás juegos lingüísticos (1985, I: 331).

\footnotetext{
${ }^{6}$ Thomas McCarthy denomina a esto postulado de igualdad dialógica: "dado que los miembros de otras culturas son necesariamente participantes en la construcción de las descripciones (accounts) que nosotros damos de ellos, si la adecuación de nuestra comprensión tiene que ser asegurada, nosotros tenemos que relacionarnos con los miembros de esas culturas de modo que reconozca la legitimidad de su derecho a responder nuestras representaciones de ellos" (Healy, 2000: 72).
} 


\section{Referencias}

Apel, K.-O. (1985), La transformación de la filosofia, 2 Tomos, Madrid: Taurus.

Apel, K.-O. y Dussel, E. (2004), Ética del discurso y ética de la liberación, Madrid: Trotta.

Bernstein, R. (1991), "Una revisión de las conexiones entre inconmensurabilidad y otredad", en Isegoría 3, 5-25.

Brandon, W. (1982), "Fact and Value in the Thought of Peter Winch: Linguistic Analysis Broaches.

Corredor, C. (1999), Filosofía del lenguaje. Una aproximación a las teorías del significado del siglo XX, Madrid: Visor.

Evans-Pritchard, E. (1937), Witchcraft, Oracles and Magic among the Azande, London: Oxford Clarendon Press.

Fernández, G. (2010) "To Understand Understanding: How Intercultural Communication is Possible in Daily Life", Human Studies 33, 371393.

Flathman, R. E. (2000), "Wittgenstein and the social sciences: critical reflections concerning Peter Winch's interpretations and appropriations of Wittgenstein`s thought", History of the Human Sciences 13(2): 1-15.

Habermas, J (1999), Teoría de la acción comunicativa. Tomo I: Racionalidad de la acción y racionalización social, Madrid: Taurus.

Healy, P. (2000), "Self-other relations and the rationality of cultures", Philosophy Social Criticism 26 (6): 61-83.

Horton, J. (2000), "Relativism, reality and philosophy", History of the Human Sciences 13(1): 19-36.

Lerner, B. D. (2002), Rules, magic and instrumental reason: a critical interpretation of Peter Winch's philosophy of social sciences, London and New York: Routledge.

Lukes, S. (2000), "Different cultures, different rationalities?", History of the Human Sciences 13(1): 3-18.

Panikkar, R. (2010), "La dialéctica de la razón armada", entrevista publicada en Topologik - Studi Filosofici 7.

Pleasants, N. (1999), Wittgenstein and the Idea of a Critical Social Theory, London and New York: Routledge.

Pleasants, N. (2000), "Winch, Wittgenstein and the Idea of a Critical Social Theory", History of the Human Sciences 13(1): 78-91.

Viaña, J. et al. (2009), Interculturalidad crítica y descolonización, La Paz: III-CAB. 
Winch, P. (1972), Ethics and Action, London: Routledge \& Kegal Paul.

Winch, P. (1990 [1958]), Ciencia social y filosofia, Buenos Aires: Amorrortu.

Winch, P. (1991), "Para comprender una sociedad primitiva", Alteridades 1(1): 82-101.

Winch, P. (1997), “Can We Understand Ourselves?”, Philosophical Investigations 20(3): 193-204.

Winch, P. (2003), The Idea of Social Sciences and it's Relation to Philosophy, $2^{\mathrm{a}}$ ed., London: Routledge. 